\title{
MC generator TAUOLA: implementation of Resonance Chiral Theory for two and three meson modes. Comparison with experiment
}

\author{
O. Shekhovtsova*, I. M. Nugent ${ }^{\dagger}$, T. Przedzinski ${ }^{* *, \sharp}$, P. Roig ${ }^{\S}$ and Z. Was ${ }^{\mathbb{I}, \sharp}$ \\ *IFIC, Universitat de València-CSIC, Apt. Correus 22085, E-46071, València, Spain ${ }^{1}$ \\ ${ }^{\dagger}$ RWTH Aachen University, III. Physikalisches Institut B, Aachen, Germany \\ ${ }^{* *}$ The Faculty of Physics, Astronomy and Applied Computer Science, \\ Jagellonian University, Reymonta 4, 30-059 Cracow, Poland \\ ${ }^{\ddagger} \mathrm{CERN} P \mathrm{PH}-\mathrm{TH}, \mathrm{CH}-1211$ Geneva 23, Switzerland \\ ${ }^{\S}$ Grup de Física Teòrica, Institut de Física d'Altes Energies, Universitat Autònoma de Barcelona, \\ E-08193 Bellaterra, Barcelona, Spain \\ II Institute of Nuclear Physics, PAN, Kraków, ul. Radzikowskiego 152, Poland
}

\begin{abstract}
We present a partial upgrade of the Monte Carlo event generator TAUOLA with the two and three hadron decay modes using the theoretical models based on Resonance Chiral Theory. These modes account for $88 \%$ of total hadronic width of the tau meson. First results of the model parameters have been obtained using BaBar data for $3 \pi$ mode.
\end{abstract}

Keywords: Tau physics, Monte Carlo event generator, chiral models

PACS: 13.35.Dx; 12.39.Fe; 29.85.-c; 89.20.Ff

\section{INTRODUCTION}

Tau lepton is the only lepton that decays into hadrons. From the perspective of low energies, as explored at B-factories, BaBar [1] and Belle [2] experiments, hadronic decay modes of tau lepton provide an excellent laboratory for modeling hadronic interactions at the energy scale of about 1-2 GeV, where neither perturbative QCD methods nor chiral lagrangians are expected to work with a good precision [3]. From the perspective of high energy experiments such as those at LHC, good understanding of tau leptons properties contributes important ingredients of new physics signatures. With the discovery of a new particle around the mass of $125-126 \mathrm{GeV}$ [4], tau decays are an important ingredient for determining if this is the Standard Model Higgs. This is especially pertinent since CMS has reported a deficit in the number of fermion decays from the new particle relative to the Standard Model Higgs Prediction.

The first version of the program TAUOLA [5] was written in the 90's. TAUOLA is Monte Carlo event generator which simulates tau decays for both the leptonic and hadronic decays modes. The hadronic currents implemented in TAUOLA are based on resonance dominance model (RDM) [6] ${ }^{2}$. In the framework of RDM the hadronic

\footnotetext{
${ }^{1}$ Speaker

2 Based on [5] Cleo and Aleph collaborations developed their own versions, non published, which differ from one another by the intermediate resonance states because of different detector sensitivity.
}

MC generator TAUOLA: implementation of Resonance Chiral Theory for two and three meson modes. Comparison with experimer 
current for a three-pseudoscalar decay mode is a sum of weighted products of the BreitWigner functions. It is demonstrated in [7] that this approach is able to reproduce only LO $\chi$ PT result. Moreover, the model was not sufficient to describe the Cleo $K K \pi$ data [8]. This resulted in the Cleo collaboration reshaping the model by introducing two ad-hoc parameters that spoilt the QCD normalization for Weiss-Zumino contribution. The parameters are obtained fitting to data. However, before making conclusion that the Wess-Zumino anomaly normalization is spoilt it should be checked whether on oversimplified theoretical approximation, like RDM, was applied.

The alternative approach based on the Resonance Chiral Theory $(\mathrm{R} \chi \mathrm{T})$ [9] was proposed in [7]. The computations done within $\mathrm{R} \chi \mathrm{T}$ are able to reproduce the lowenergy limit of $\chi \mathrm{PT}$ at least up to NLO and demonstrate the right falloff in the high energy region [10]. The hadronic currents for the two and three meson modes $(\pi \pi, K \pi$, $K K, \pi \pi \pi$ and $K K \pi$ ) calculated in the framework of $\mathrm{R} \chi \mathrm{T}$ were implemented in TAUOLA [11]. The tar-ball version of the code is available at web-page [12].

\section{RESULTS FOR TWO AND THREE MESON FINAL STATES}

It is of utmost importance to implement the theory in a form that is as useful for general applications as possible. Therefore the corresponding hadronic currents $\left(J^{\mu}\right)$ in the upgraded version of TAUOLA have been written in the most general form compatible with Lorentz invariance. For $\tau$ decay channels with two mesons $\left(h_{1}\left(p_{1}\right)\right.$ and $\left.h_{2}\left(p_{2}\right)\right)$

$$
J^{\mu}=N\left[\left(p_{1}-p_{2}-\frac{\Delta_{12}}{s}\left(p_{1}+p_{2}\right)\right)^{\mu} F^{V}(s)+\frac{\Delta_{12}}{s}\left(\left(p_{1}+p_{2}\right)^{\mu} F^{S}(s)\right],\right.
$$

where $\Delta_{12}=m_{1}^{2}-m_{2}^{2}, s=\left(p_{1}+p_{2}\right)^{2}$. For the final state of three pseudoscalars, with momenta $p_{1}, p_{2}$ and $p_{3}$, Lorentz invariance determines the decomposition

$$
\begin{aligned}
J^{\mu}=N \quad & \left\{T_{v}^{\mu}\left[c_{1}\left(p_{2}-p_{3}\right)^{v} F_{1}+c_{2}\left(p_{3}-p_{1}\right)^{v} F_{2}+c_{3}\left(p_{1}-p_{2}\right)^{v} F_{3}\right]\right. \\
& \left.+c_{4} q^{\mu} F_{4}-\frac{i}{4 \pi^{2} F^{2}} c_{5} \varepsilon_{. v \rho \sigma}^{\mu} p_{1}^{v} p_{2}^{\rho} p_{3}^{\sigma} F_{5}\right\},
\end{aligned}
$$

where: $T_{\mu \nu}=g_{\mu \nu}-q_{\mu} q_{v} / q^{2}$ denotes the transverse projector, $q^{\mu}=\left(p_{1}+p_{2}+p_{3}\right)^{\mu}$ is the momentum of the hadronic system, $F$ is the pion decay constant in the chiral limit.

The decomposition (1) and (2) is the most general one, model-dependence is included in the hadronic form factors $\left(F_{V}, F_{S}\right.$ as well as $\left.F_{i}, \mathrm{i}=1 \ldots 5\right)$. The hadronic form factors calculated within $\mathrm{R} \chi \mathrm{T}$ can be written as

$$
F_{I}=F_{I}^{\chi}+F_{I}^{R}+F_{I}^{R R}
$$

where $F_{I}^{\chi}$ is the chiral contribution, $F_{I}^{R}$ is the one resonance contribution and $F_{I}^{R R}$ is the double-resonance part. The explict form of the functions $F_{i}$ for $3 \pi$ and $K K \pi$ modes can be found in [11], Section 2, as well as in [7, 13]. The theoretical results for the hadronic currents were obtained in the isospin limit $\left(m_{\pi}=\left(2 m_{\pi^{+}}+m_{\pi^{0}}\right) / 3\right.$, $\left.m_{K}=\left(m_{K^{+}}+m_{K^{0}}\right) / 2\right)$, except for the two pion and two kaons modes. In the phase space generation, the differences between neutral and charged pion and kaon masses is 
TABLE 1. The $\tau$ decay partial widths. The PDG value [14] (2nd collumn) is compared with numerical results from TAUOLA with the $\mathrm{R} \chi \mathrm{T}$ currents: in the isospin limit for pseudoscalar masses (3rd collumn), using physical masses (4th collumn).

\begin{tabular}{|c|c|c|c|}
\hline \multirow{2}{*}{ Channel } & \multicolumn{3}{|c|}{ Width, [GeV] } \\
\cline { 2 - 4 } & PDG & Equal masses & $\begin{array}{c}\text { Phase space } \\
\text { with masses }\end{array}$ \\
\hline$\pi^{-} \pi^{0}$ & $(5.778 \pm 0.35 \%) \cdot 10^{-13}$ & $(5.2283 \pm 0.005 \%) \cdot 10^{-13}$ & $(5.2441 \pm 0.005 \%) \cdot 10^{-13}$ \\
$K^{-} \pi^{0}$ & $(9.72 \pm 3.5 \%) \cdot 10^{-15}$ & $(8.3981 \pm 0.005 \%) \cdot 10^{-15}$ & $(8.5810 \pm 0.005 \%) \cdot 10^{-15}$ \\
$\pi^{-} \bar{K}^{0}$ & $(1.9 \pm 5 \%) \cdot 10^{-14}$ & $(1.6798 \pm 0.006 \%) \cdot 10^{-14}$ & $(1.6512 \pm 0.006 \%) \cdot 10^{-14}$ \\
$K^{-} K^{0}$ & $(3.60 \pm 10 \%) \cdot 10^{-15}$ & $(2.6502 \pm 0.007 \%) \cdot 10^{-15}$ & $(2.6502 \pm 0.008 \%) \cdot 10^{-15}$ \\
$\pi^{-} \pi^{-} \pi^{+}$ & $(2.11 \pm 0.8 \%) \cdot 10^{-13}$ & $(2.1013 \pm 0.016 \%) \cdot 10^{-13}$ & $(2.0800 \pm 0.017 \%) \cdot 10^{-13}$ \\
$\pi^{0} \pi^{0} \pi^{-}$ & $(2.10 \pm 1.2 \%) \cdot 10^{-13}$ & $(2.1013 \pm 0.016 \%) \cdot 10^{-13}$ & $(2.1256 \pm 0.017 \%) \cdot 10^{-13}$ \\
$K^{-} \pi^{-} K^{+}$ & $(3.17 \pm 4 \%) \cdot 10^{-15}$ & $(3.7379 \pm 0.024 \%) \cdot 10^{-15}$ & $(3.8460 \pm 0.024 \%) \cdot 10^{-15}$ \\
$K^{0} \pi^{-} \bar{K}^{0}$ & $(3.9 \pm 24 \%) \cdot 10^{-15}$ & $(3.7385 \pm 0.024 \%) \cdot 10^{-15}$ & $(3.5917 \pm 0.024 \%) \cdot 10^{-15}$ \\
$K^{-} \pi^{0} K^{0}$ & $(3.60 \pm 12.6 \%) \cdot 10^{-15}$ & $(2.7367 \pm 0.025 \%) \cdot 10^{-15}$ & $(2.7711 \pm 0.024 \%) \cdot 10^{-15}$ \\
\hline
\end{tabular}

taken into account, i.e. physical values are chosen. This has to be done to obtain proper kinematic configurations. The model parameters, more specifically the masses of the resonances and the coupling constants, were fitted to Aleph data, requiring correct highenergy behaviour of the related form factors, see Appendix C in [11].

To check stability of multidimensional integration in TAUOLA the MC results were compared with the semi-analytical ones (Gauss integration of analytical results was used). The difference between MC prediction and semi-analytical results for the partial decay width is less than $0.02 \%$ for all channels. Both differential spectra and numerical tests are collected at [12].

In Table 1, the partial decay width values from PDG [14] are compared with our results obtained with isospin-averaged pseudoscalar masses and with the physical ones. Comparison of the last two columns illustrates numerical effect of physical masses. The difference between the MC result and PDG one is $2 \%-24 \%$ depending on the channel. As expected, that agreement is not good because only minimal attempts on adjusting to the model parameters have been applied for the comparison with BaBar and Belle data. The next section presents the first step toward this direction.

\section{FIT FOR THREE PION MODE TO BABAR DATA}

The main problem with upgrading the MC simulation of $\tau$ decays is a lack of the published spectrum. Currently, only the two pion modes [2] and three pseudoscalar modes [15] are published.

The results from a preliminary fit to the three pion mode $\left(\pi^{+} \pi^{-} \pi^{-}\right)$[15] can be seen in Fig. 1 and Table 2. Both the three particle and the $\pi^{+} \pi^{-}$invariant mass distributions have been considered. Disagreement about $12 \%$ level is visible in the low energy region of two particle invariant mass distribution whereas for the three invariant mass spectrum the difference between $\mathrm{MC}$ and data is less than $7 \%$. However, the $\mathrm{R} \chi \mathrm{T}$ parametrization is in better agreement with BaBar data than CLEO one, see Fig. 1.

The fit was done taking into account only $P$-wave contribution of two pion system. 
TABLE 2. Numerical values of the $\mathrm{R} \chi \mathrm{T}$ parameters fitted to $\mathrm{BaBar}$ data for three pion mode

\begin{tabular}{|l|l|l|l|l|l|l|l|}
\hline & $M_{\rho^{\prime}}$ & $\Gamma_{\rho^{\prime}}$ & $M_{a_{1}}$ & $F$ & $F_{V}$ & $F_{A}$ & $\beta_{\rho^{\prime}}$ \\
\hline Min. & 1.44 & 0.32 & 1.00 & 0.0920 & 0.12 & 0.1 & -0.36 \\
\hline Max & 1.48 & 0.39 & 1.24 & 0.0924 & 0.24 & 0.2 & -0.18 \\
\hline Default & 1.453 & 0.4 & 1.12 & 0.0924 & 0.18 & 0.149 & -0.25 \\
\hline Fit ${ }^{*}$ & 1.4302 & 0.376061 & 1.21706 & 0.092318 & 0.121938 & 0.11291 & -0.208811 \\
\hline
\end{tabular}

* to[15], $\chi^{2} / n d f=2262.12 / 132$
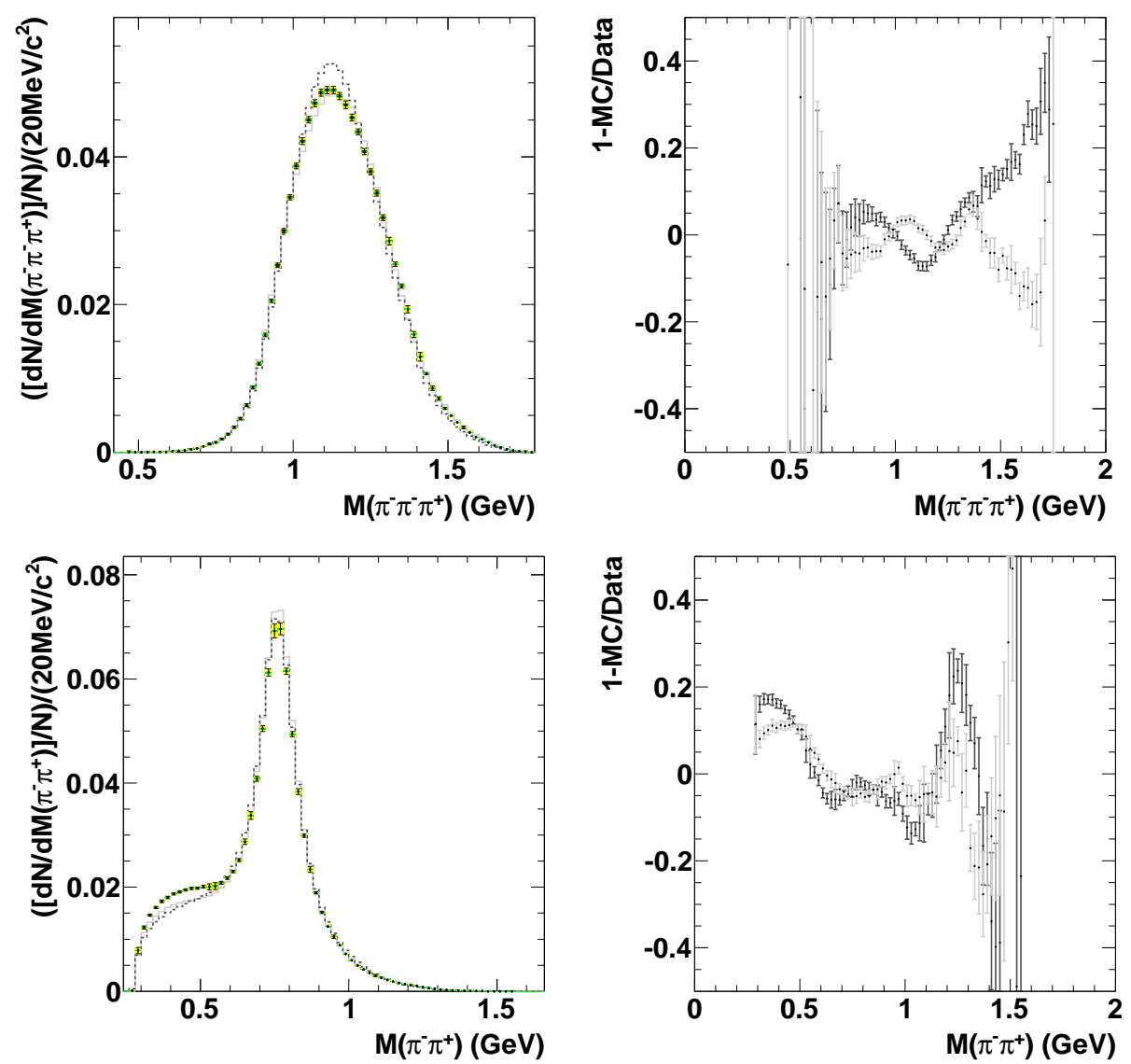

FIGURE 1. Invariant $\pi^{+} \pi^{-} \pi^{-}$(up) and $\pi^{+} \pi^{-}$(down) mass distributions: the plots on the left-hand side correspond to the differential decay distribution, the ones on the right-hand side to plot ratios between $\mathrm{MC}$ and $\mathrm{BaBar}$ data. Lighter grey histograms are for $\mathrm{R} \chi \mathrm{T}$ parametrization, darker grey is for CLEO one.

As suggesting in [16] the discrepance in the low mass region could be described using a contribution from the scalar particle, $S$-wave contribution. We expect that inclusion of $S$-wave contribution [10] will improve the value of $\chi^{2}$. The values in the fifth row of Table 2 are only the preliminary results. They will not necessarily correspond to the minimum of $\chi^{2} / n d f$ of the final fit. Work is in progress.

MC generator TAUOLA: implementation of Resonance Chiral Theory for two and three meson modes. Comparison with experimer 


\section{CONCLUSION}

The theoretical results for the hadronic currents of two and three pseudoscalar modes, namely, $\pi \pi, K \pi, K K, \pi \pi \pi$ and $K K \pi$, in the framework of $\mathrm{R} \chi \mathrm{T}$ have been implemented in TAUOLA. These modes, together with the one-meson decay modes, represent more than $88 \%$ of the hadronic width of tau. $\mathrm{R} \chi \mathrm{T}$ is a more controlled QCD-based model than the usual used Breit-Wigner parametrizations. However, before making conclusion about validity of the model the theoretical results have to be confronted with the experimental data, which requires fit of the model parameters. Now that the technical work on current installation is complete, the work on fits is in progress in collaboration with theoreticians and experimentalists.

At LHC tau decays are only used for identification and are not used to study their dynamic. However, the dynamics of tau decays are important for both modeling the decays and -therefore the reconstruction and identification- and for measuring the polarization of tau decays. Therefore, an upgrade to the TAUOLA based on the BaBar and Belle results on tau decays is urgently needed for LHC.

\section{ACKNOWLEDGMENTS}

This research was supported by a Marie Curie Intra European Fellowship within the 7th European Community Framework Programme (O.S.) and by Alexander von Humboldt Foundation (I.N.), by the Spanish Consolider Ingenio 2010 Programme CPAN (CSD2007-00042) and by MEC (Spain) under Grants FPA2007-60323, FPA2011-23778 (O.S. and P.R.) and FPA2011-25948 (P.R.) and in part by the funds of Polish National Science Centre under decision DEC-2011/03/B/ST2/00107 (T.P., Z.W.).

\section{REFERENCES}

1. B. Aubert et al., Phys.Rev.Lett. 100 (2008) 011801; J.P. Lees et al., arXiv: 1109.1527 [hep-ex].

2. M. Fujikawa et al., Phys. Rev. D78 (2008) 072006; H. Hayashii, M. Fujikawa, Nucl.Phys.Proc.Suppl. 198 (2010) 157.

3. E. Braaten and C.-S. Li, Phys.Rev. D42 (1990) 3888.

4. G. Aad et al., arXiv: 1207.7214 [hep-ex]; S. Chatrchyan, arXiv:1207.7235 [hep-ex].

5. S. Jadach, Z. Wa̧s, R. Decker and J. H. Kühn, Comput. Phys. Commun. 76 (1993) 361.

6. J.H. Kühn, E. Mirkes, Z. Phys. C56 (1992) 661; J.H. Kühn, A. Santamaría, Z. Phys. C48 (1990) 445.

7. D. G. Dumm, A. Pich and J. Portolés, Phys. Rev. D69 (2004) 073002.

8. T.E. Coan et al., Phys.Rev.Lett. 92 (2004) 232001.

9. G. Ecker, J. Gasser, H. Leutwyler, A. Pich, E. de Rafael, Phys. Lett. B223 (1989) 425; Nucl. Phys. B321 (1989) 311.

10. P. Roig, I. Nugent, T. Przedzinski, O. Shekhovtsova, Z. Was, in these Proceedings.

11. O. Shekhovtsova, T. Przedzinski, P. Roig and Z. Was, arXiv: 1203.3955 [hep-ph].

12. T. Przedzinski, O. Shekhovtsova and Z. Was, http://annapurna.ifj.edu.pl/ wasm/RChL/RChL.htm.

13. D.G. Dumm, P. Roig, A. Pich, J. Portolés, Phys. Lett. B685 (2010) 158; Phys. Rev. D81 (2010) 034031.

14. K. Nakamura, J. Phys. G37 (2010) 075021.

15. I.M. Nugent, SLAC-R-936.

16. E.I. Shibata et al., arXiv: hep-ex/0210039.

MC generator TAUOLA: implementation of Resonance Chiral Theory for two and three meson modes. Comparison with experimer 